\title{
Flaring Rate Distribution of Gamma-Ray Blazars and Implications for High-Energy Neutrino Emission
}

\author{
Kenji Yoshida* \\ Shibaura Institute of Technology, Saitama, Japan \\ E-mail: yoshida@shibaura-it.ac.jp
}

\section{Maria Petropoulou ${ }^{\dagger}$}

Princeton University, Princeton NJ, USA

\section{Meg Urry, Paolo Coppi, Charles Bailyn, Georgios Vasilopoulos}

Yale University, New Haven CT, USA

\section{Kohta Murase}

The Pennsylvania State University, University Park PA, USA

\section{Foteini Oikonomou}

European Southern Observatory, Garching bei München, Germany

\begin{abstract}
Gamma-ray flares from blazars have been suggested as ideal periods for the detection of highenergy neutrinos. In fact, the first $\sim 3 \sigma$ high-energy neutrino source association was based on the detection of a single neutrino (IC-170922A) coincident with the flaring blazar TXS 0506+056. To better understand the contribution of gamma-ray flares to the blazar neutrino output, a statistical study of the flare properties is needed. Using public data from the Fermi Monitored Source List for 142 bright blazars, we construct a sample with the fraction of time spent in the flaring state (i.e. the flare duty factor) and the fraction of energy released during its flare. We predict the neutrino output of each source during gamma-ray flares using a theoretically motivated relation between neutrino and gamma-ray luminosities (i.e. the neutrino luminosity is proportional to the gamma-ray luminosity to the power of $1.5-2.0$ ). We use the quiescent X-ray flux of blazars, as determined from public Swift-XRT data, to estimate the neutrino flux during non-flaring states. By comparing our results with the IceCube sensitivity for week-long transients, we constrain the standard leptonic scenario for the blazar gamma-ray emission, and provide the upper-limit contribution of gamma-ray flares of bright blazars to the isotropic neutrino background.
\end{abstract}

36th International Cosmic Ray Conference -ICRC2019-

July 24th - August 1st, 2019

Madison, WI, U.S.A.

\footnotetext{
${ }^{*}$ Speaker.

${ }^{\dagger}$ MP acknowledges support from the Lyman Spitzer, Jr. Postdoctoral Fellowship and from the Fermi Guest Investigator grant $80 \mathrm{NSSC} 18 \mathrm{~K} 1745$.
} 


\section{Introduction}

Blazars are the most active class of active galactic nuclei (AGNs) with very rapid variability, high/variable polarization, superluminal motion, and very high luminosity. They are divided into Flat-Spectrum Radio Quasars (FSRQs) and BL Lac objects (BL Lacs) [1]. In a blazar, it is believed that the relativistic jet from the central supper-massive black hole is oriented along the line of sight. Blazars have been discussed as the sources of ultrahigh-energy cosmic rays and/or high-energy neutrinos (e.g. [2, and references therein]).

Recently, IceCube Collaboration et al. (2018) reported a sub-PeV muon neutrino event in 2017, IceCube-170922A, which was coincident in direction and time with a gamma-ray flare of blazar TXS 0506+056 at the statistical significance level of $3 \sigma$ [3]. Furthermore, independent of and prior to the 2017 flaring event, IceCube Collaboration (2018) reported $3.5 \sigma$ evidence for neutrino events from the direction of TXS 0506+056 with an archival search of past IceCube data [4]. The IceCube Collaboration has also observed high-energy astrophysical neutrinos in the $100 \mathrm{TeV}-\mathrm{PeV}$ range that are consistent with an isotropic arrival distribution [5]. The isotropic high-energy muon neutrino flux from the northern sky with IceCube is found to be $E_{v}^{2} \Phi_{v}(E)=1.0 \times 10^{-8} \mathrm{GeV} \mathrm{cm}^{-2} \mathrm{~s}^{-1} \mathrm{sr}^{-1}$ [6]. The isotropic neutrino background can be regarded as neutrinos produced by a large number of extra-galactic sources (e.g. [7]).

In this paper, we present the flaring rate distribution of gamma-ray blazars and estimate the high-energy neutrino emission of blazar flares. We also discuss the contribution of flares of bright gamma-ray blazars to the high-energy diffuse neutrino emission.

\section{Gamma-ray Data Analysis}

\subsection{Data Set}

We use the weekly-binned and decade-long gamma-ray light curves of 142 blazars in the energy range of 0.1-300 GeV, which are taken from the public Monitored Source List data by Fermi-LAT [8]. The sample consists of 100 FSRQs, 32 BL Lacs, and 10 blazars of uncertain type. Although the data are produced by using preliminary instrument response functions and calibrations, the comparison of several public light curves with light curves of the same sources analyzed by using the latest Pass 8 instrumental response functions and the P8R2 SOURCE event selection [9] indicates that our key results are robust against uncertainties of the preliminary analysis.

\subsection{Quiescent Flux Level}

Although there is no rigorous way to define the quiescent level of blazar emission, it is reasonable to assume that the quiescent state has a relatively low and stable flux. In this study, we adopted the following procedure to derive the quiescent level:

1. We applied a Bayesian blocks algorithm to each gamma-ray light curve with the false alarm probability $p_{0}=0.075[10,11]$. The Bayesian blocks algorithm provides the partitions of the light curve into blocks, whose fluxes are regarded to be constant during the partitioned interval. 


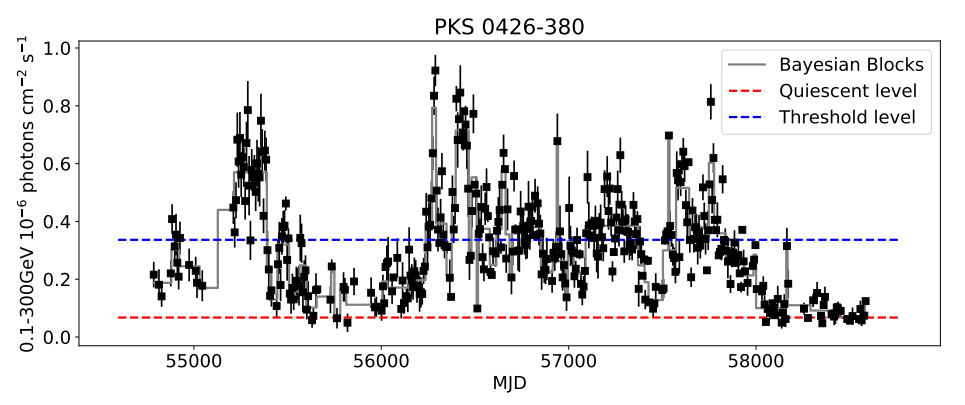

Figure 1: An example of a weeklybinned blazar gamma-ray light curve (black points) with the quiescent and flare threshold $(6 \sigma)$ levels indicated with dashed red and blue lines, respectively. The light-curve representation with Bayesian Blocks is also shown (gray solid lines).

2. We computed the average flux of each Bayesian block, which represents an optimal stepfunction light curve.

3. We derived the minimum block flux as the quiescent level, on the condition that the number of data points of the block is more than or equal to the average number of data points of the blocks. This condition prevents us from using an insufficient amount of data points to estimate the quiescent level.

Figure 1 shows as an indicative example the weekly-binned Fermi-LAT light curve of PKS 0426380 (black points) and the Bayesian Blocks representation (solid gray lines). The quiescent flux level derived using the method described above is also indicated with a dashed red line.

\subsection{Flaring Threshold Level}

If the flux of the weekly-binned measurements exceeds a certain threshold, then this is defined as a gamma-ray flare flux. The threshold $F_{\gamma}^{t h}$ is given by

$$
F_{\gamma}^{t h}=F_{\gamma}^{q}+s\left\langle F_{\gamma}^{e r r}\right\rangle
$$

where $F_{\gamma}^{q}$ is the gamma-ray quiescent level, $\left\langle F_{\gamma}^{e r r}\right\rangle$ is an average error of the gamma-ray light curve, and $s$ corresponds to the significance above the quiescent level in units of the standard deviation $\sigma$. Unless otherwise noted, we use $s=6$ in this work. Figure 1 also shows an example of the flaring threshold level of the PKS 0426-380 gamma-ray light curve.

\subsection{Flare Duty Factor and Flare Energy Fraction}

The fraction of time spent in the flaring state, i.e. the flare duty factor, is given by

$$
f_{f l}=\frac{1}{T_{t o t}} \int_{F_{\gamma}^{t h}} d F_{\gamma} \frac{d T}{d F_{\gamma}}
$$

where $T_{\text {tot }}$ is the total observation time. The fraction of energy emitted in the flaring state, i.e. the gamma-ray flare energy fraction, is given by

$$
b_{f l}^{\gamma}=\frac{1}{F_{\gamma}^{\text {ave }} T_{\text {tot }}} \int_{F_{\gamma}^{t h}} d F_{\gamma} F_{\gamma} \frac{d T}{d F_{\gamma}}
$$

where $F_{\gamma}^{a v e}$ is the average gamma-ray flux over the whole observation period.

Figure 2 presents the distributions of the flare duty factors and flare energy fractions for the $0.1-300 \mathrm{GeV}$ gamma-ray light curves of 124 blazars, which produced gamma-ray flares above the threshold. Their distributions are well represented by a power-law function with the index of $-0.97 \pm 0.05$ and $-1.03 \pm 0.07$, respectively. 

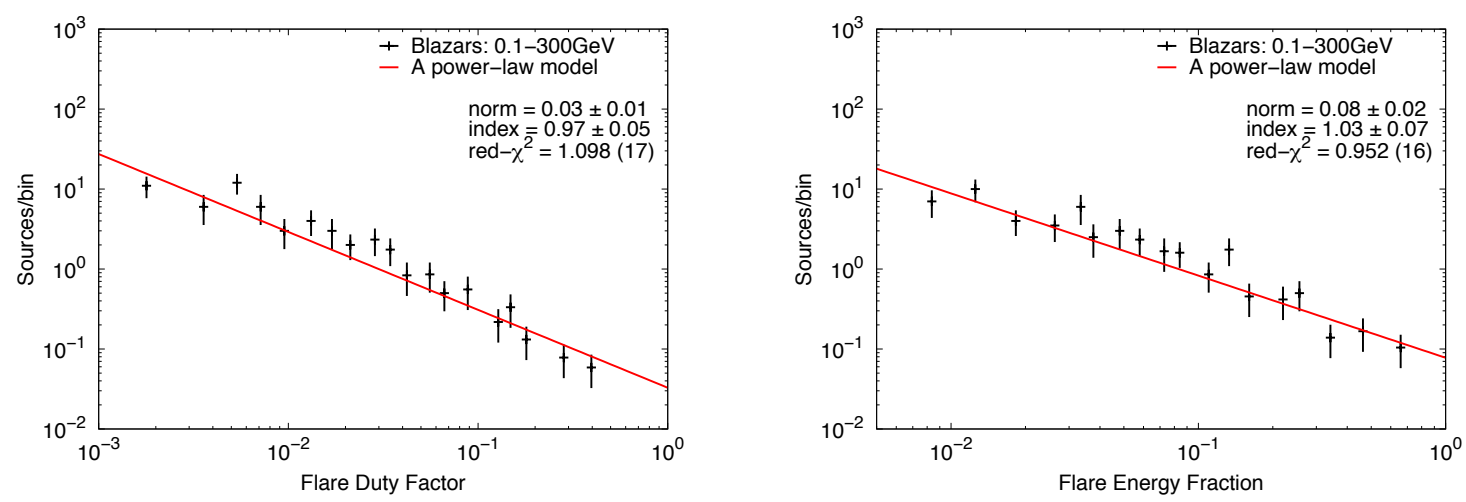

Figure 2: Distributions of the $0.1-300 \mathrm{GeV}$ gamma-ray flare duty factors (left panel) and flare energy fractions (right panel) of 124 blazars. The red lines are best-fit power-law functions to each distribution.

\section{Implication for High-Energy Neutrino Emission}

\subsection{Estimation of Muon Neutrino Flux}

In the standard leptonic or hadronic scenario for the blazar gamma-ray emission, flares can dominate the neutrino output of a blazar. Different models of blazar gamma-ray emission predict that the neutrino flux is related to the gamma-ray flux as: $F_{v} \propto F_{\gamma}^{\gamma}$, where the index $\gamma$ ranges from 1.5 to 2.0 [7, and references therein]. If one assumes $F_{V} \propto F_{\gamma}$, high-energy neutrino emission may be dominated by the non-flaring contribution. On the other hand, the X-ray quiescent level in $0.3-10 \mathrm{keV}$ band places a rough upper limit to the muon neutrino quiescent flux in the $100 \mathrm{TeV}$ - PeV range (for details, see [12, and references therein]). In particular, we assume that the Xray quiescent flux is equal to the muon neutrino quiescent flux. For the X-ray data, we use Open Universe for Blazars, which provides us blazar X-ray light curves based on 14 years of Swift-XRT data [13]. The X-ray quiescent level is derived in a similar way to the gamma-ray quiescent level (see section 2.2). The muon neutrino flare flux, $\varepsilon_{v} F_{v_{\mu}}^{f l}$, is then estimated as

$$
\varepsilon_{v} F_{v_{\mu}}^{f l}=\varepsilon_{X} F_{X}^{q}\left(\frac{F_{\gamma}^{f l}}{F_{\gamma}^{q}}\right)^{\gamma}
$$

where $\varepsilon_{X} F_{X}^{q}$ is the X-ray quiescent level in units of $\mathrm{erg} \mathrm{cm}^{-2} \mathrm{~s}^{-1}$.

Figure 3 shows an example of the time history of the muon neutrino fluxes estimated for the nearby blazar Mrk 421 using gamma-ray and X-ray data. As shown in Fig. 3, our results, which are obtained using a simple scaling relation between the gamma-ray and neutrino flux, and therefore are independent of the details of neutrino production in this source, are in quantitative agreement with those of the theoretical model presented in [14] ${ }^{1}$. The IceCube Collaboration reported the results of the search for steady point-like sources in the astrophysical muon neutrino flux with 8 years of IceCube data [15]. We compared our results with the IceCube $5 \sigma$ discovery potential with an $E_{v}^{-2}$ power-law neutrino spectrum, which is scaled to the sensitivity for a week after multiplying

\footnotetext{
${ }^{1}$ In [14], Petropoulou et al. (2016) demonstrated the role of major gamma-ray flares in the neutrino output of Mrk 421. Here, we also find the maximum neutrino enhancement during the same flaring periods.
} 


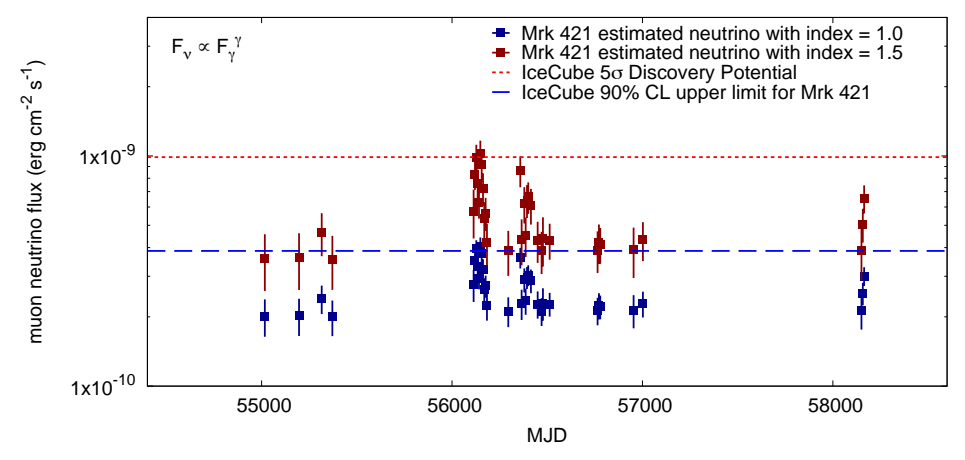

Figure 3: An example of time history of muon neutrino flux with $\gamma=$ 1.0 and $\gamma=1.5$ (Mrk 421). The blue dashed line shows the IceCube $90 \%$ CL upper limit for Mrk 421 and the red dotted line shows the IceCube $5 \sigma$ discovery potential (both are scaled to a week) [15].

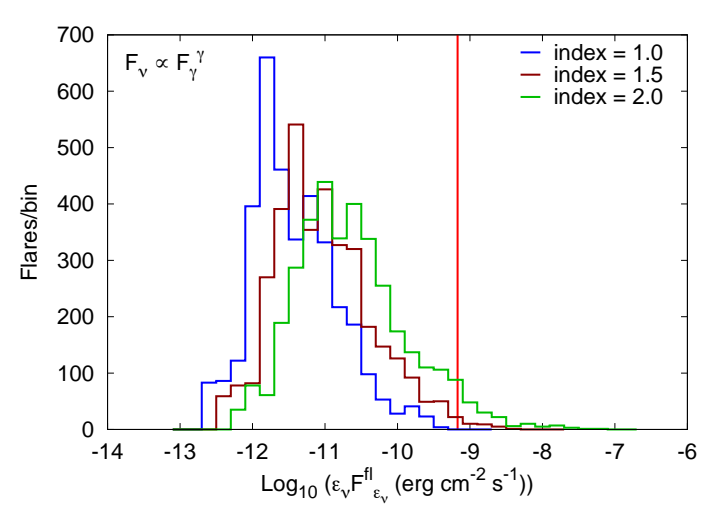

Figure 4: Estimated neutrino flare flux distribution from 124 gamma-ray blazars with $\gamma=1.0, \gamma=$ 1.5 , and $\gamma=2.0$. The vertical red line corresponds to an IceCube $8 \mathrm{yr}$ typical discovery potential of $1.0 \times 10^{-12} \mathrm{TeV} \mathrm{cm}^{-2} \mathrm{~s}^{-1}$ (scaled to a week).

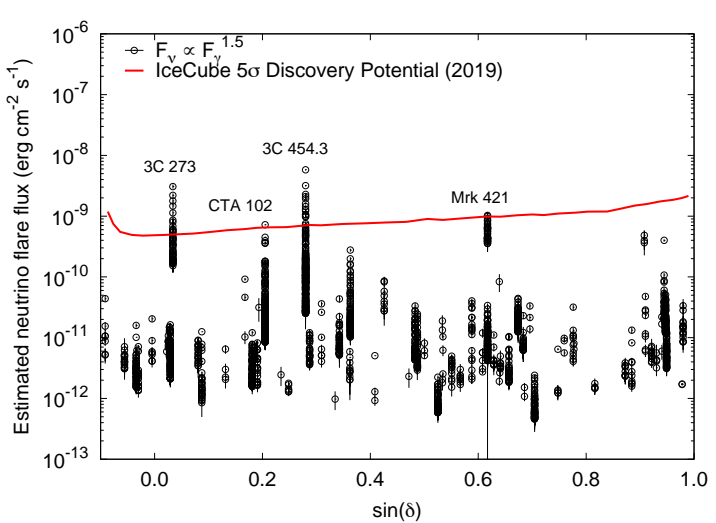

Figure 5: Estimated muon neutrino flare fluxes with $\gamma=1.5$ as a function of the $\sin (\delta)$ with declination $\delta$. The IceCube $5 \sigma$ discovery potential (scaled to a week) is also plotted for an $E_{v}^{-2}$ neutrino spectrum (red solid).

with the factor $8.0 \times 365.0 / 7.0$. As shown in Fig. 3, the peak flare fluxes of Mrk 421 with $\gamma=1.5$ are close to or above the IceCube $5 \sigma$ potential discovery, but those with $\gamma=1.0$ are lower than the IceCube discovery potential [15].

Figure 4 shows the flare flux distributions of the estimated muon neutrino with indices $\gamma=1.0$, $\gamma=1.5$, and $\gamma=2.0$. The vertical line corresponds to the typical IceCube discovery sensitivity of $1.0 \times 10^{-12} \mathrm{TeV} \mathrm{cm}^{-2} \mathrm{~s}^{-1}$ for $8 \mathrm{yr}$, which is scaled to a week. As shown in Fig. 4, there are no neutrino flares above the sensitivity for $\gamma=1.0$, but neutrino flares of several sources with $\gamma=1.5$ and $\gamma=2.0$ lie above the sensitivity limit.

In order to compare our results with IceCube $5 \sigma$ discovery potential in more detail, we plot the estimated muon neutrino flare fluxes with $\gamma=1.5$ as a function of the $\sin (\delta)$ with declination $\delta$ (Fig. 5). The IceCube $5 \sigma$ discovery potential is scaled to a week. As shown in Fig. 5, the neutrino flare fluxes predicted for 3C 273, CTA 102, 3C 454.3, and Mrk 421 are close to or above the $5 \sigma$ discovery potential.

\subsection{Contribution of Blazar Flares to the Isotropic Neutrino Background}

In the case of the standard leptonic scenario for the blazar gamma-ray emission of $F_{v} \propto F_{\gamma}^{\gamma}$ with $\gamma=1.5-2.0$, the neutrino flare energy fraction, $b_{f l}^{v}$, becomes more enhanced than the gamma- 
ray flare energy fraction. Figure 6 presents the distribution of the neutrino flare energy fractions with $\gamma=1.5$. The distribution is well represented by a power-law function with the index of $-0.57 \pm 0.06$, which shows a flatter distribution than that of the gamma rays (see the right panel in Fig. 2). The distribution of the neutrino flare energy fractions with $\gamma=2.0$ gives a much flatter power-law distribution, which has an index of $-0.28 \pm 0.11$. This suggests that the neutrino output of blazars becomes increasingly dominated by flares as $\gamma$ becomes larger.

By using the redshifts of the blazars taken from [16, 17], we derived the neutrino flare luminosities from the flare fluxes: $\varepsilon_{v} L_{v}^{f l}=4 \pi d_{L}^{2} \varepsilon_{v} F_{v}^{f l}$, where $d_{L}$ is a luminosity distance with $\Omega_{m 0}=0.308, \Omega_{\Lambda 0}=0.692$, and $H_{0}=67.8 \mathrm{~km} \mathrm{~s}^{-1} \mathrm{Mpc}^{-1}$ [18]. Figure 7 shows the neutrino flare luminosity distributions obtained for $\gamma=1.0, \gamma=1.5$, and $\gamma=2.0$.

In order to estimate the contribution of blazar flares to the isotropic neutrino background, we follow [7], and in particular, we use their eq. (7) of [7]. We set the solid angle covered by the IceCube detector to be $\Delta \Omega=2 \pi$ and adopt the IceCube typical $5 \sigma$ discovery potential for a point source with an $E_{v}^{-2}$ neutrino spectrum of $1.0 \times 10^{-12} \mathrm{TeV} \mathrm{cm}^{-2} \mathrm{~s}^{-1}$ for $8 \mathrm{yr}$, which is scaled to a week to be $F_{\text {lim }}=6.7 \times 10^{-10} \mathrm{erg} \mathrm{cm}^{-2} \mathrm{~s}^{-1}$. We also adopt a luminosity-dependent correction factor determined by the redshift evolution $q_{L}=1$ and a factor of the multiplet analysis $b_{m}=6.6$ for $m \geq 2$. As a result, the contribution of blazar flares to the isotropic neutrino background is constrained as

$$
E_{v}^{2} \Phi_{v} \lesssim 3.8 \times 10^{-10} \mathrm{GeVcm}^{-2} \mathrm{~s}^{-1} \mathrm{sr}^{-1}\left(\frac{\xi_{z}}{0.7}\right)\left(\frac{0.05}{f_{f l}}\right)^{1 / 2}\left(\frac{b_{f l}^{v}}{1.0}\right)^{1 / 2}\left(\frac{10^{46} \mathrm{ergs}^{-1}}{\varepsilon_{v} L_{\varepsilon_{v_{\mu}}}^{f l}}\right)^{1 / 2},
$$

where $\xi_{z}$ is a dimensionless parameter that depends on the redshift evolution of the sources: $\xi_{z}=$ 0.68 for the gamma-ray luminosity density evolution of BL Lacs and $\xi_{z}=8.4$ for that of FSRQs [19, and references therein].

The contribution of flares from bright gamma-ray blazars (like those of our sample) to the isotropic neutrino background can be estimated by summing up the individual contributions and then divided by their number, i.e. an average of the upper-limit values of eq.(3.2) over all flares. Figure 8 shows the distribution of the upper-limit values of $E_{v}^{2} \Phi_{v}$ from the individual flares with $\gamma=1.5$. For the flaring bright blazars of this sample, the averages of the upper-limit values of the isotropic neutrino background are $(1.43 \pm 0.08) \times 10^{-9} \mathrm{GeV} \mathrm{cm}^{-2} \mathrm{~s}^{-1} \mathrm{sr}^{-1}$ for $\gamma=1.0$, $(1.20 \pm 0.08) \times 10^{-9} \mathrm{GeV} \mathrm{cm}^{-2} \mathrm{~s}^{-1} \mathrm{sr}^{-1}$ for $\gamma=1.5$, and $(0.96 \pm 0.08) \times 10^{-9} \mathrm{GeV} \mathrm{cm}^{-2} \mathrm{~s}^{-1} \mathrm{sr}^{-1}$ for $\gamma=2.0$. They correspond to $14 \%, 12 \%$, and $10 \%$ of the isotropic neutrino background, respectively. As described in [7], fainter blazar flares could make a larger contribution.

So far, we have defined gamma-ray flares with the $6 \sigma$ level $(s=6)$ above the quiescent gammaray flux. Figure 9 presents the averages of the upper-limit values of $E_{v}^{2} \Phi_{v}$ as a function of the flare significance $s$ above the quiescent level with $\gamma=1.5$. As shown in Fig. 9, the estimated contribution of blazar flares to the isotropic neutrino background is almost independent of the significance $s$ (down to the level of $4 \sigma$ ).

\section{Discussion and Conclusions}

As presented in Fig. 5, some neutrino flares of 3C 273, CTA 102, 3C 454.3, and Mrk 421 estimated with $\gamma=1.5$ exceed the IceCube $5 \sigma$ discovery potential in the $\sin \delta$ range from -0.1 to 


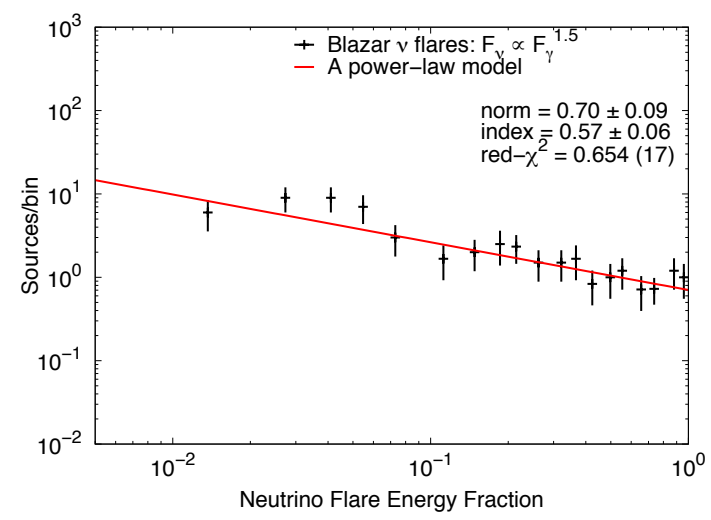

Figure 6: Distribution of neutrino flare energy fraction with $\gamma=1.5$. The best-fitted power-law function is also plotted (red line).

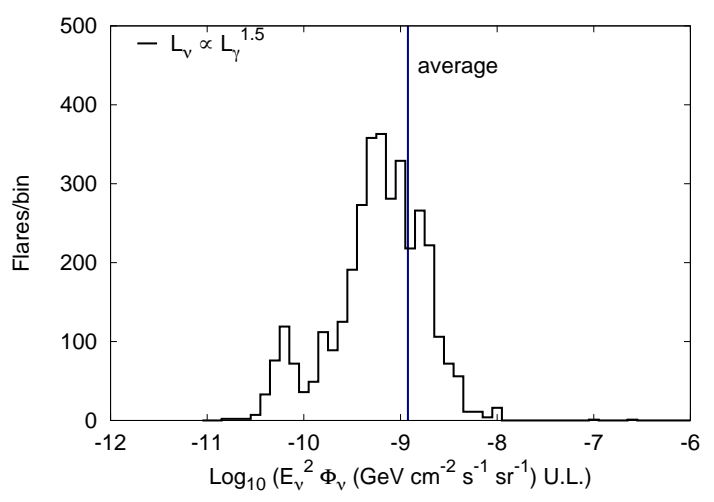

Figure 8: Distribution of the upper limits of $E_{v}^{2} \Phi_{v}$ from the individual flares for $\gamma=1.5$. The blue vertical line corresponds to the average over the flares.

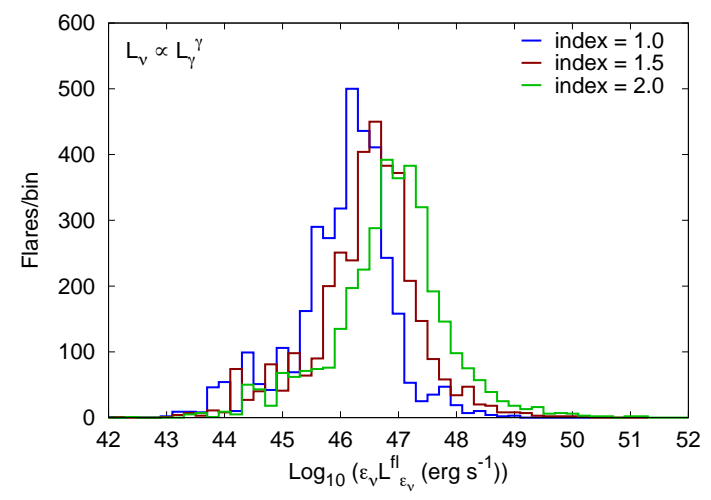

Figure 7: Estimated neutrino flare luminosity distributions with indices $\gamma=1.0$ (blue), $\gamma=1.5$ (dark red), and $\gamma=2.0$ (green),

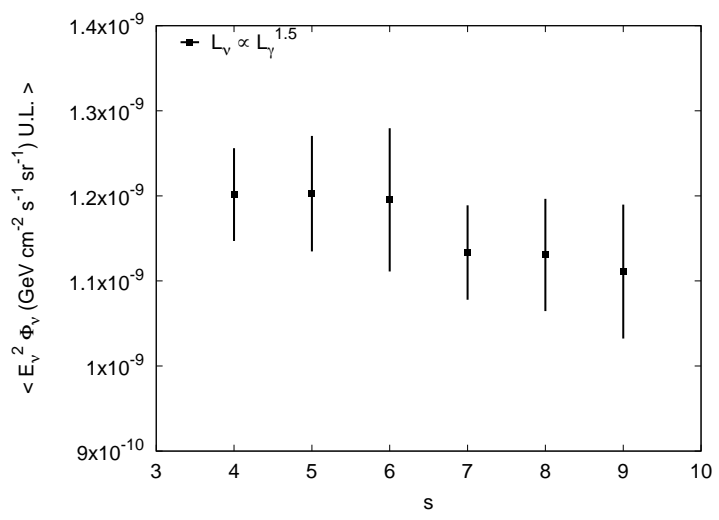

Figure 9: The averages of $E_{v}^{2} \Phi_{v}$ U.L. as a function of the flare significance $s$ above the quiescent level with $\gamma=1.5$.

1.0 [15]. In the case of $\gamma=2.0$, the predicted number of neutrino flares exceeding the IceCube potential becomes even higher. The lack of neutrino detection from these sources suggests that either the neutrino quiescent level is much lower than the X-ray quiescent level or the index $\gamma$ is much smaller than 2.0 .

So far, in all the neutrino estimates, we have assumed a flat neutrino spectrum. Alternatively, we can compute the expected number of neutrinos from 3C 273, 3C 454.3, and Mrk 421 using theoretical model spectra with a peak energy typically lying beyond $1 \mathrm{PeV}[14,20,15]$, while taking into account the time-dependent IceCube effective area and exposure source by source. For this calculations, we assumed that the model spectra represent the quiescent flux, and normalized the model spectra by dividing the quiescent neutrino flux and multiplying the estimated neutrino flux with $\gamma=1.5$. Figure 10 represents the time history of the expected number of muon neutrinos of 3C 273, 3C 454.3, and Mrk 421. As shown in Fig. 10, the expected number of neutrinos from these sources is almost below the IceCube $5 \sigma$ discovery potential. This is due to the neutrino model spectral shape, which results in fewer neutrinos at and below $1 \mathrm{PeV}$ than a flat $E_{v}^{-2}$ spectrum. In 


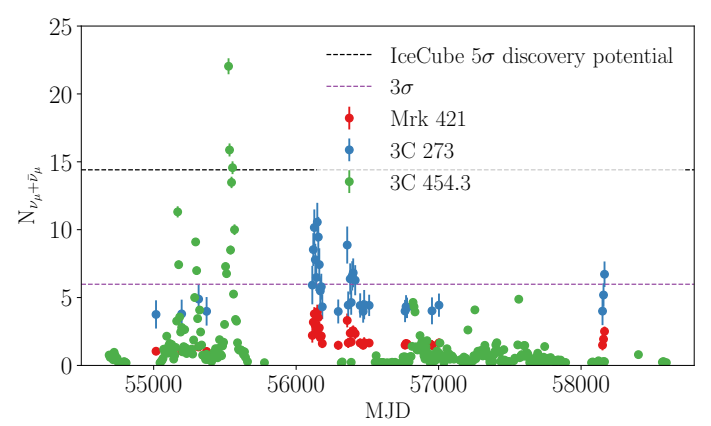

Figure 10: Time histories of the expected number of muon neutrinos of 3C 273 (sky blue), 3C 454.3 (green), and Mrk 421 (red) with IceCube $5 \sigma$ and $3 \sigma$ discovery potentials. They are calculated from the model spectra in the quiescent state $[14,20,15]$ with our results of the estimated flaring neutrino fluxes for $\gamma=1.5$.

addition, the IceCube exposure gets worse with increasing energy. In agreement with our neutrino flux estimation with $\gamma=1.5$, for these sources, we can rule out models that have a soft neutrino spectrum peaking at lower energies than $1 \mathrm{PeV}$ more readily.

The IceCube $5 \sigma$ discovery potential constrains our estimation of the muon neutrino fluxes of the flaring blazars. Although flares from dimmer gamma-ray blazars could make a larger contribution to the isotropic neutrino background, we can already place an upper limit of $12-14 \%$ to the contribution of bright gamma-ray flaring blazars, in accordance with the stacking/clustering constraints derived in [7, and references therein].

\section{References}

[1] C.M. Urry \& P. Padovani, PASP 107, 803 (1995).

[2] K. Murase, arXiv:1511.01590v2 (2016).

[3] The IceCube Collaboration et al., Science 361, 146 (2018).

[4] The IceCube Collaboration, Science 361, 1472013151 (2018).

[5] The IceCube Collaboration, Phys. Rev. Lett. 113, 101101 (2014).

[6] The IceCube Collaboration, Phys. Rev. Lett. 115, 081102 (2015).

[7] K. Murase, F. Oikonomou, \& M. Petropoulou, Astrophys. J. 865, 124 (2018).

[8] http://fermi.gsfc.nasa.gov/ssc/data/policy/LAT_Monitored_Sources.html

[9] M. Meyer, J.D. Scargle, \& R.D. Blandford, arXiv:1902.02291v1 (2019)

[10] J.D. Scargle, et al., Astrophys. J. 764, 167 (2013).

[11] The Astropy Collaboration, Astron. J. 156, 123 (2018).

[12] P. Padovani et al., MNRAS 484, L104 (2019).

[13] P. Giommi et al. arXiv:1904.06043v1 (2019).

[14] M. Petropoulou, S. Coenders, \&S. Dimitrakoudis, Astropart. Phys. 80, 115 (2016).

[15] The IceCube Collaboration, arXiv:1811.07979v2 (2019).

[16] M. Ackermann et al. arXiv:1501.06054v2 (2015).

[17] http://ned.ipac.caltech.edu/

[18] M. Tanabashi et al. (Particle Data Group), Phys. Rev. D 98, 030001 (2018).

[19] K. Murase \& E. Waxman, Phys. Rev. D 94, 103006 (2016).

[20] A. Reimer, PoS ICRC 2015, 1123 (2015). 